\title{
Passive Nonlinear Pulse Shaping in Normally Dispersive Fiber Systems
}

\author{
Sonia Boscolo, Anton I. Latkin, and Sergei K. Turitsyn
}

\begin{abstract}
We propose a novel approach to characterize the parabolically-shaped pulses that can be generated from more conventional pulses via nonlinear propagation in cascaded sections of commercially available normally dispersive (ND) fibers. The impact of the initial pulse chirp on the passive pulse reshaping is examined. We furthermore demonstrate that the combination of pulse pre-chirping and propagation in a single ND fiber yields a simple, passive method for generating various temporal waveforms of practical interest.
\end{abstract}

Index Terms-Nonlinear fiber optics, nonlinear pulse propagation, passive pulse shaping, parabolic pulses.

\section{INTRODUCTION}

$\mathbf{N}$ ONLINEAR phenomena in optical fibers can be exploited to develop new techniques for regeneration, processing and manipulation of the optical signals. Recent developments in nonlinear optics have put into the focus of intensive research an interesting class of pulses with a parabolic profile in the energy-containing core and a linear (in time) frequency chirp that can propagate in a fiber with normal group-velocity dispersion (GVD) [1]-[7]. While in the normal dispersion regime nonlinear-dominated optical pulse broadening generally leads to wave breaking which manifests itself as waveform steepening with subsequent growing oscillations at the pulse tails (see, e.g., [1], [8], [9] and references therein), parabolic pulses (PPs) propagate in a stable self-similar manner, holding certain relations (scaling) between changing pulse power, width, and chirp parameter. Because of their unique characteristics, parabolic pulses are of great interest for various applications including amongst others high-power femtosecond lasers, spectral broadening and supercontinuum generation, and nonlinear all-optical signal processing and regeneration. The problem of the PP generation in fibers has attracted a great deal of attention in recent years [7]. PPs can be generated through the asymptotic reshaping of sufficently powerful pulses that occurs upon propagation in normally dispersive (ND), nonlinear fiber amplifying media. Experimental demonstrations relying on amplification from either rare-earth doping [2], [3] or Raman scattering [5] have confirmed the potential of this method, especially when dealing with the generation of ultrashort, high-power pulses. However, in the context of applications where high signal power is not necessarily required and the most valuable features are the specific parabolic shape and phase of the

S. Boscolo and S. K. Turitsyn are with the Photonics Research Group, School of Engineering and Applied Science, Aston University, Birmingham B4 7ET, U.K. (e-mail: s.a.boscolo@aston.ac.uk). A. I. Latkin is with the Novosibirsk State University, Novosibirsk, 630090, Russia. pulse, such as, e.g., optical telecommunications, other approaches to the PP generation could be of interest. To this end, several techniques have been recently proposed: the use of a dispersion-decreasing fiber [10]-[13], the application of superstructured fiber Bragg gratings [14], and the combination of two carefully chosen ND fibers [15].

In this paper, we present an accurate analysis of the nonlinear reshaping of conventional pulses that occurs upon propagation in cascaded sections of commercially available ND fibers. In many respects, our analysis follows and generalizes the pioneering work of [15], where a very important and simple method of generating linearly chirped PPs in a complete passive manner has been proposed. Compared to previous techniques, the approach suggested in [15] has the clear advantage of relaxing the need to use an amplifying medium (and thus the need for a pumping source), or the need for additional custom devices [14]. Indeed, it relies only on the use of commercially available fibers with normal dispersion. Here, we extend the results of [15] in several directions. We characterize in detail the evolution of an initial Gaussian pulse into a pulse with a nearly parabolic temporal intensity profile and a nearly linear chirp during propagation in a ND fiber, and the subsequent pulse stabilization in a second ND fiber with suitably different nonlinear and dispersive characteristics relative to the first fiber. We discuss the tolerance of the PP formation to the length of the initial reshaping fiber as well as the impact of the initial pulse chirp on the pulse reshaping process. We furthermore propose the combination of pulse pre-chirping and propagation in a single ND fiber as a simple method for passive nonlinear pulse shaping in the time domain. Techniques for the control and manipulation of the temporal shape of optical pulses have become increasingly important for many scientific applications, including, ultrahigh-speed optical telecommunications and computing systems, quantum optics, and nonlinear optics. Here, we demonstrate that by means of control of the initial chirp and power, conventional laser pulses can be transformed into pulses with various temporal waveforms of practical interest, ranging from a parabolic profile to a flat-top profile, and a triangular profile. Flat-top pulses are highly desired for nonlinear optical signal processing and switching as well as for a range of wavelength conversion applications. Triangular pulses are also of interest, e.g., for time-domain add-drop multiplexing, wavelength conversion and other photonic applications.

\section{SYSTEM DESCRIPTION AND PULSE SHAPING MODELING}

The proposed pulse reshaper consists of a pre-chirping device, an optical amplifier, and a section of ND fiber, as 


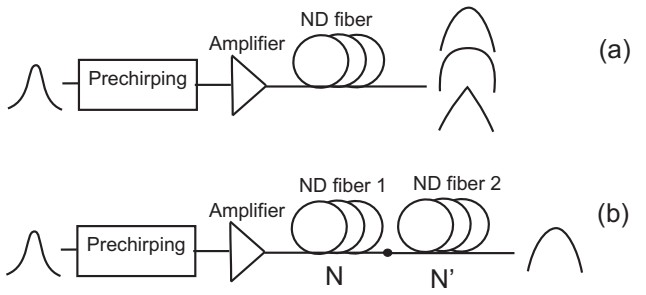

Fig. 1. Scheme of the pulse reshaper. (a) One-stage device, (b) two-stage device.

depicted in Fig. 1(a). Qualitatively, the idea for the method is as follows: a transform-limited (chirp-free) optical pulse generated from a laser source incoming to the reshaper is first subject to the preferred chirping. Then the pulse is amplified by the optical amplifier to enhance the effect of nonlinearity in the ND fiber. During transmission along the ND fiber, the temporal waveform of the pulse can be changed to various profiles by the combined action of GVD and Kerr nonlinearity according to the chirping value and power level at the input of the fiber. Note that the newly formed pulses in the device have all a nearly linear chirp (first time derivative of the phase). It is also worth noting that such pulse shapes represent transient states of the nonlinear pulse evolution in a passive ND fiber. This is in contrast with the self-similar PPs that can be obtained as asymptotic, approximate solutions of the nonlinear Schrödinger equation (NLSE) in a ND fiber amplifier in the large-amplitude/small-dispersion limit [3], [4], [6], [7]. Finally, we point out that the generation of PPs through passive pulse reshaping in a ND fiber has been already demonstrated in [15], and different types of pulse temporal waveforms have been achieved in [16] using a two cascaded interferometers' reshaping architecture. The approach proposed here generalizes the method proposed in [15], offering additional flexibility in the generation of the target waveform by control of the pulse pre-chirping.

Our numerical simulations of the optical pulse propagation in fiber are based on the standard NLSE model including the effects of fiber dispersion, nonlinearity and, when explicitely indicated, absorption [17]. To simplify the analysis, we use the dimensionless quantities: $u(\xi, \tau)=N U, U(\xi, \tau)=$ $\psi / \sqrt{P_{0}}, \tau=t / T_{0}$ and $\xi=z / L_{\mathrm{D}}$. Here, $\psi(z, t)$ is the field envelope in the comoving system of coordinates, $T_{0}$ and $P_{0}$ are respectively a characteristic temporal width (e.g., the halfwidth at $1 / e$-intensity point in the case of a Gaussian-shaped pulse) and the peak power of the initial pulse, and $L_{\mathrm{D}}, L_{\mathrm{NL}}$ and $N$ are respectively the dispersion length, the nonlinear length and the energy parameter ("soliton" number) defined as: $L_{\mathrm{D}}=T_{0}^{2} / \beta_{2}, L_{\mathrm{NL}}=1 /\left(\gamma P_{0}\right)$ and $N=\sqrt{L_{\mathrm{D}} / L_{\mathrm{NL}}}$, where $\beta_{2}$ and $\gamma$ are the respective GVD and nonlinearity parameters of the fiber. For the purpose of illustration, we consider the propagation of an intial Gaussian-shaped pulse: $U(0, \tau)=\exp \left(-\tau^{2} / 2+i C \tau^{2}\right)$ in a highly nonlinear (HNL) fiber. Propagation in the fiber is considered up to a maximum length of $L_{\mathrm{D}} . C$ is the (normalized) chirp parameter. Note that for a different choice of the initial pulse shape, similar pulse shaping regimes to those reported in this paper are expected to occur upon propagation in a ND fiber, whereas the relevant parameter regions would be different [15]. In order to quantify the evolution of the initial pulse towards a pulse with some shape and a linear chirp, we introduce dimensionless functionals defined as

$$
\begin{gathered}
\kappa=\frac{\int_{-\infty}^{\infty} \mathrm{d} \tau \tau^{2}|u|^{2}\left(\int_{-\infty}^{\infty} \mathrm{d} \tau|u|^{4}\right)^{2}}{\left(\int_{-\infty}^{\infty} \mathrm{d} \tau|u|^{2}\right)^{5}}, \\
\tilde{\kappa}=\frac{\int_{-\infty}^{\infty} \mathrm{d} \omega \omega^{2}|\tilde{u}|^{2}\left(\int_{-\infty}^{\infty} \mathrm{d} \omega|\tilde{u}|^{4}\right)^{2}}{\left(\int_{-\infty}^{\infty} \mathrm{d} \omega|\tilde{u}|^{2}\right)^{5}}, \\
\Phi=\frac{\int_{-\Delta \tau / 2}^{\Delta \tau / 2} \mathrm{~d} \tau|| \phi_{\tau \tau}|-|\left(\phi_{\tau \tau}\right)_{\tau=0}||}{\int_{-\Delta \tau}^{\Delta \tau} \mathrm{d} \tau\left|\left(\phi_{\tau \tau}\right)_{\tau=0}\right|} .
\end{gathered}
$$

In (1), the structural functional $\kappa$ characterizes the pulse temporal shape [18], [19]. For example, $\kappa=0.0796$ for a Gaussian pulse, $\kappa=0.072$ for an ideal PP corresponding to the intensity profile: $\left|u_{\mathrm{P}}(\tau)\right|^{2}=1-\left(\tau / \tau_{\mathrm{P}}\right)^{2}$ if $|\tau| \leq$ $\tau_{\mathrm{P}}$ and $\left|u_{\mathrm{P}}(\tau)\right|^{2}=0$ otherwise, and $\kappa=0.0741$ for an ideal triangular pulse defined as $\left|u_{\mathrm{T}}(\tau)\right|^{2}=1-\left|\tau / \tau_{\mathrm{T}}\right|$ if $|\tau| \leq \tau_{\mathrm{T}}$ and $\left|u_{\mathrm{T}}(\tau)\right|^{2}=0$ otherwise. Obviously, $\kappa$ is not the only possible choice of dimensionless quantity that can be used for shape characterization. Nonetheless the form of this coefficient provides the simplest way of describing the pulse shape in terms of meaningful physical characteristics of the pulse. Indeed, one may notice that $\kappa=\left(T_{\text {int }} P_{\text {int }} / E\right)^{2}$, where $T_{\text {int }}=\left(\int \mathrm{d} \tau \tau^{2}|u|^{2} / E\right)^{1 / 2}$ and $P_{\text {int }}=\int \mathrm{d} \tau|u|^{4} / E$ are the second-order moments related to the pulse temporal width and power, respectively, [19], [20] and $E=\int \mathrm{d} \tau|u|^{2}$ is the pulse energy. In [19], $\kappa$ has been associated with an invariant of the propagation equations of the second-order moments of the pulse amplitude and effective power within the parabolic approximation for the pulse phase. This invariant indirectly expresses the conservation of energy. Since it is normalized by the energy, which is also an invariant, it describes the pulse shape. In (1), $\tilde{\kappa}$ is an analogous functional defined for the pulse spectral shape, where $\omega$ is the (normalized) frequency and $\tilde{u}$ is the Fourier transform of the field envelope. $\phi=\arg u$ is the phase of the field, and parameter $\Phi$ gives a measure of the linearity of the frequency chirp across a time interval $\Delta \tau$ around $\tau=0$ such that $\int_{-\Delta \tau / 2}^{\Delta \tau / 2} \mathrm{~d} \tau|u|^{2}=a \int_{-\infty}^{\infty} \mathrm{d} \tau|u|^{2}$, where $0<a \leq 1$. In other words, $\Phi$ quantifies the misfit between the slope of the frequency chirp at some $\tau$ and the slope at $\tau=0$ in the energy-containing central portion of the pulse, which is parametrized by $a$. For the purpose of comparison, we will also consider in our analysis the misfit parameter $M$ between the pulse temporal intensity profile and a parabolic fit of the same energy and full-width at half-maximum duration used in [15]:

$$
M^{2}=\frac{\int_{-\infty}^{\infty} \mathrm{d} \tau\left(|u|^{2}-\left|u_{\mathrm{P}}\right|^{2}\right)^{2}}{\int_{-\infty}^{\infty} \mathrm{d} \tau|u|^{4}} .
$$

Following [15], it is possible to stabilize the PP shape generated in a length of ND fiber by launching the pulses into a second fiber such that the soliton number $N^{\prime}$ is higher than the soliton number $N$ in the initial reshaping fiber: $N^{\prime} / N>1$. Here, $N^{\prime}$ is defined by $N^{\prime 2}=T_{0}^{2} \gamma^{\prime} P_{0} / \beta_{2}^{\prime}$, where $\beta_{2}^{\prime}$ and $\gamma^{\prime}$ are the parameters of the second fiber, and $T_{0}$ and $P_{0}$ relate to the pulse parameters at the input of the first fiber. The 
schematic diagram of the two-stage fiber device is depicted in Fig. 1(b). As an illustrative example, we choose to use a fiber with nonlinearity and dispersive characteristics such that $\gamma^{\prime} / \beta_{2}^{\prime}=9 \gamma / \beta_{2}$. With this respect, we would like to mention that an alternate approach to increase $N^{\prime}$ relative to $N$ is to amplify the pulses before propagation in the second fiber (in such case $N^{\prime}$ is defined by: $N^{\prime 2}=T_{0}^{2} \gamma^{\prime} G P_{0} / \beta_{2}^{\prime}$, where $G$ is the gain between the two fibers), whereas this approach is not completely passive and might bring about perturbation of the pulse due to amplification. The total length of the two-stage system is set to $4 L_{\mathrm{D}}$. Such (not optimized) length yields a reasonable compromise between the quality of the parabolic pulses obtainable at the system output and the system complexity.

The dimensionless analysis performed in this paper enables us to obtain results that can be applied to a variety of situations in the real world's units by simple scaling of the relevant quantities. A target operational regime and output pulse shape can be achieved here by changing $\xi, N$ and $C$. In practical situations, it is usually the case that one has a pulse source with a given pulse duration $T_{0}$, and a ND fiber with given dispersive and nonlinear characteristics, $\beta_{2}$ and $\gamma$. Therefore, to achieve the desired operational regime and pulse shape one simply needs to change the fiber length $z$, the input pulse peak power $P_{0}$ (or input pulse energy) and the input chirp parameter $C / T_{0}^{2}$.

\section{PARABOLIC PULSE GENERATION IN A NORMALLY DISPERSIVE TWO-SEGMENT FIBER DEVICE}

In this section, we study the progressive nonlinear reshaping of an initial pulse into a pulse with a close-to-ideal parabolic shape and a nearly linear chirp in a ND fiber, and the subsequent shape-maintaining propagation of the pulse in a further piece of fiber.

Figure 2 shows the evolution of the relative temporal and spectral shape factors $K$ and $\tilde{K}$, the misfit parameter $M$, and the phase factor $\Phi$ versus the length of the first fiber section ( $\xi$ ranging from 0 to 1 ) and the energy parameter $N$ (from 1 to 10) for an initial Gaussian pulse with $C=0$. Here, $K$ and $\tilde{K}$ are defined as: $K=\left(\kappa-\kappa_{\mathrm{P}}\right) / \kappa_{\mathrm{P}}$, and $\tilde{K}=\left(\tilde{\kappa}-\tilde{\kappa}_{\mathrm{P}}\right) / \tilde{\kappa}_{\mathrm{P}}$, where $\kappa_{\mathrm{P}}=\tilde{\kappa}_{\mathrm{P}}=0.072$, and $a$ is set to 0.95 . It is seen that parameters $K$ and $M$ exhibit similar trends, confirming the results of [15]. The initial pulse energy, or in other words the value of $N$ strongly influences the pulse reshaping to a parabolic form. The optimum distance $\xi_{\text {opt }}$ where $K(M)$ reaches a minimum decreases with increasing $N$, and the higher the value of $N$, the faster the increase of $K(M)$ after $\xi_{\text {opt }}$, indicating that the parabolic temporal intensity profile is more difficult to maintain. This represents a very different behaviour compared to that of self-similar PPs which maintain their intensity profile during amplification [3]. We can also see in Fig. 2 that $N$ influences $K_{\mathrm{opt}} \equiv K\left(\xi_{\mathrm{opt}}\right)\left(M_{\mathrm{opt}}\right)$ and that an optimum $N$ exists: $N_{\text {opt }} \sim 2.5-4$ (moderate energies). The absolute minimum of $K$ (corresponding to $\kappa_{\mathrm{opt}} \approx 0.0723$ ) occurs here at $\left(N_{\text {opt }}=2.8, \xi_{\text {opt }}=0.446\right) . M$ is at its absolute minimum $\left(M_{\mathrm{opt}} \approx 0.033\right)$ for $\left(N_{\mathrm{opt}}=2.6, \xi_{\mathrm{opt}}=0.413\right)$. It is worth noting that the distance at which the pulse becomes
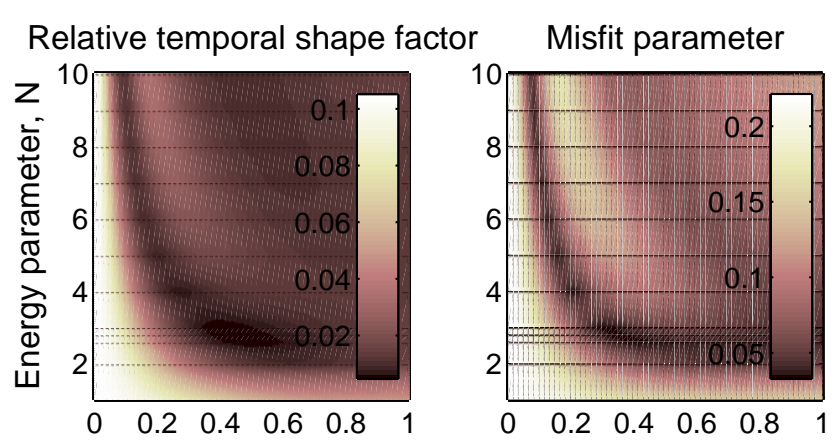

Relative spectral shape facto

Phase factor
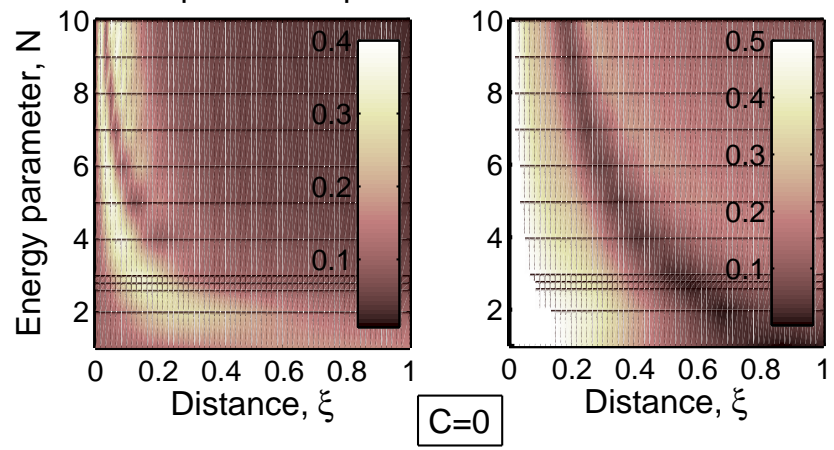

Fig. 2. First-stage evolution of the relative temporal and spectral shape factors $K$ and $\tilde{K}$, the misfit parameter $M$, and the phase factor $\Phi$ (for $a=0.95$ ) versus $\xi$ and $N$ for $C=0$.

parabolic precedes the onset of wave breaking [15]. Figure 2 also shows that the phase factor has a similar qualitative behavior as the temporal shape factor. On the other hand, the band of operating conditions for which $\Phi$ is very low is shifted towards the region of larger distances compared with the band where the pulse temporal profile is nearly parabolic. Moreover, $\Phi$ takes its absolute minimum value for $N=1$ (low energies) or, in other words when the pulse evolution in the fiber is almost linear. Again, such a behavior constitutes a major difference compared to the asymptotic generation of a self-similar PP which leads to a perfectly linear chirp [3]. However, it is evident from Fig. 2 that there exists a relatively large region $(N, \xi)$ around the optimum point $\left(N_{\text {opt }}, \xi_{\text {opt }}\right)$ for $K(M)$ where the frequency chirp is sufficiently linear $(\Phi<0.1)$ over the majority of the pulse.

Figures 3 and 4 show the pulse temporal and spectral intensity profiles and the chirp profile at the output of the first and second fiber segments obtained from numerical integration of the NLSE, when the pulses are launched into the second fiber at the optimum distance $\xi_{\text {opt }}$ and for the optimum energy $N_{\text {opt }}$ for which $K$ and $M$ reach their minima in the intial reshaping fiber, respectively. It can be seen that after propagation in the first stage, the pulse temporal profile is already very close to the desired parabolic shape, as confirmed by the good agreement between the temporal intensity distribution and the parabolic fit (of the same energy and FWHM width), and the chirp (instantaneous frequency) is linear over the energycontaining part of the pulse. On the other hand, the spectrum of the pulse remains substantially Gaussian-shaped - as the initial pulse spectrum is - during propagation in the first stage 

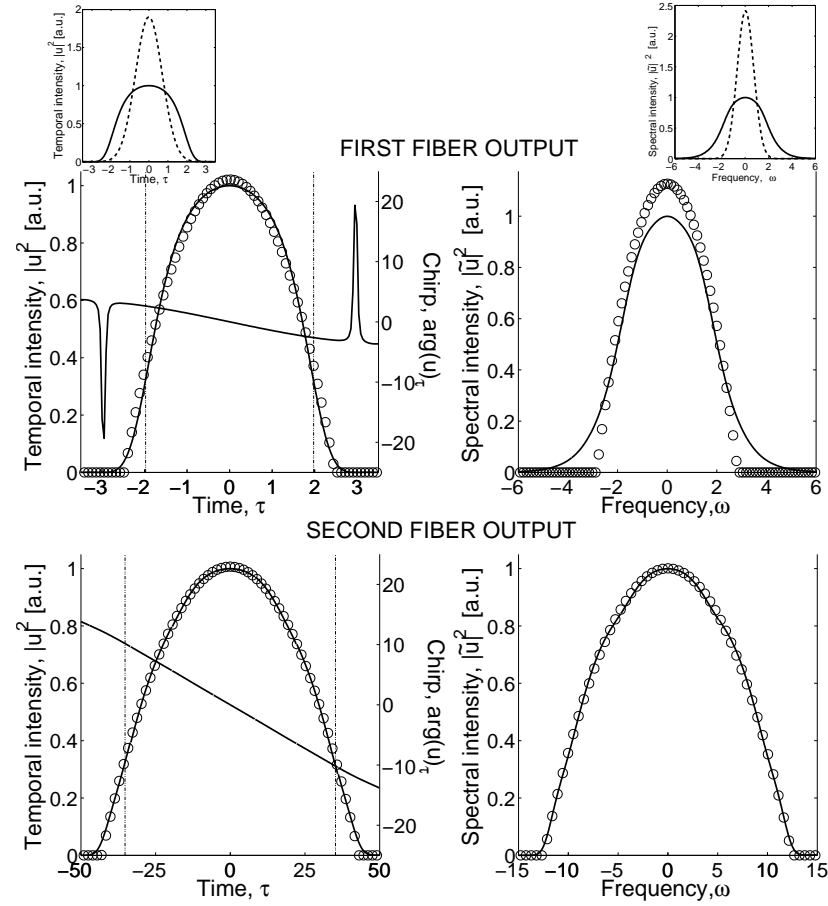

Fig. 3. Pulse temporal and spectral intensity profiles and chirp profile at the input and output of the second stage starting at $\xi=0.446$ for $N=2.8$. Solid curves: NLSE numerical simulation results, circles: parabolic fits. The vertical lines mark the boundaries of the region $\Delta \tau$ where $a=0.95$. Insets: Intensity profiles at the input of the first stage (dashed lines) and second stage (solid lines).
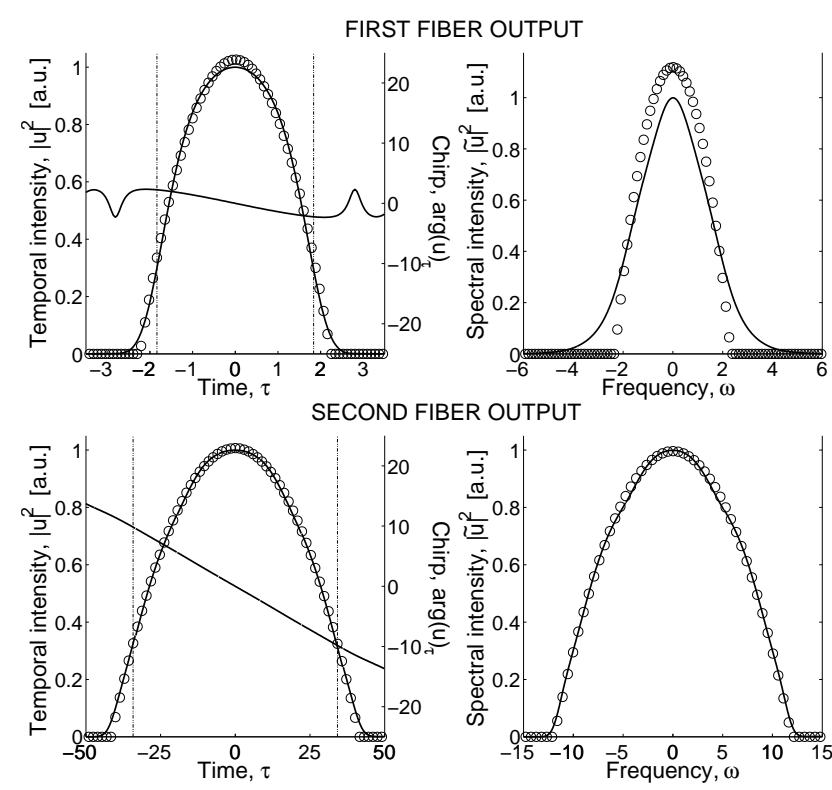

Fig. 4. Pulse temporal and spectral intensity profiles and chirp profile at the input and output of the second stage starting at $\xi=0.413$ for $N=2.6$. Solid curves: NLSE numerical simulation results, circles: parabolic fits. The vertical lines mark the boundaries of the region $\Delta \tau$ where $a=0.95$.

ending at the optimum $\xi$ for $M$, while the beginning of a spectral reshaping of the pulse is observable at the output of the first stage ending at the optimum $\xi$ for $K$. In any case, at this stage the spectrum does not have the parabolic intensity profile characteristic of a highly chirped PP [1], indicating relatively moderate nonlinear effects during the first-stage pulse evolution. The situation is different at the output of the second stage. There, the temporal intensity profile has stabilized to an almost ideal parabolic shape and the chirp is highly linear over the entire pulse duration. Moreover, a large degree of spectral broadening and reshaping is observed which can be fitted well with a parabolic shape. The PPs generated in the first stage are thus seen to undergo large self-phase modulation-induced spectral broadening without evidence of any oscillations in the wings and concomitant side lobes in the spectrum characteristic of wave breaking [1]. These results are in agreement with the results reported in [15]. Moreover, the comparison between Figs. 3 and 4 indicates that starting the second stage at the point $\left(N_{\text {opt }}, \xi_{\text {opt }}\right)$ of either minimum $K$ or minimum $M$ yields similar results in terms of degree of parabolicity of the pulse shape in the temporal and spectral domains and of linearity of the frequency chirp. Here and here after, we will use parameter $K$ as an indicator of the pulse shaping process.
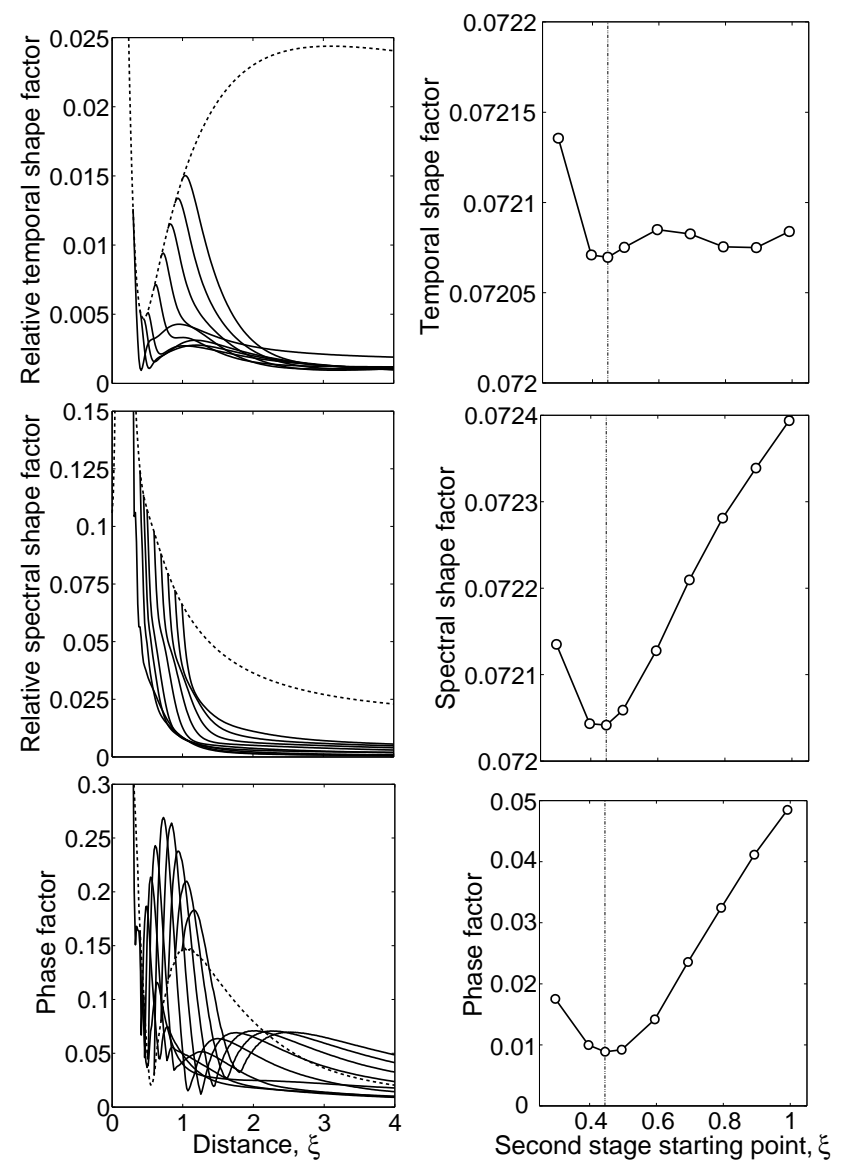

Fig. 5. Left graphs, evolutions of the relative temporal and spectral shape factors $K$ and $\tilde{K}$, and the phase factor $\Phi$ versus $\xi$ in the second fiber, for $N=2.8$ and the second stage starting at different $\xi$. Dashed curves, corresponding evolutions over a single-stage fiber device for $N=2.8$. Right graphs, temporal and spectral intensity factors $\kappa$ and $\tilde{\kappa}$ and phase factor at the output of the second stage as a function of the second stage starting point.

Next, we investigate the tolerance of the PP generation in the two-stage fiber system to the length of the initial reshaping fiber segment. The left graphs in Fig. 5 show the evolutions of the relative temporal and spectral shape factors and the phase 

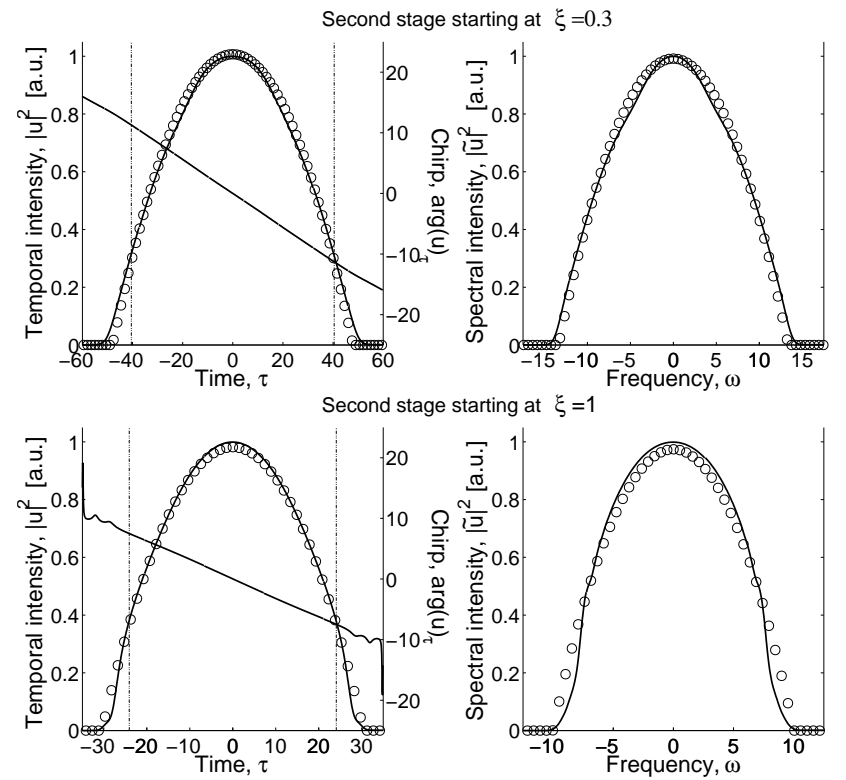

Fig. 6. Pulse temporal and spectral intensity profiles and chirp profile at the output of the second stage starting at $\xi=0.3$ and $\xi=1$ for $N=2.8$. Solid curves: NLSE numerical simulation results, circles: parabolic fits. The vertical lines mark the boundaries of the region $\Delta \tau$ where $a=0.95$.

factor over the distance in the second fiber, when the second stage starts at different $\xi$ and the energy parameter is set at the optimum value of $N_{\text {opt }}=2.8$ relative to $K$ in the first fiber. The corresponding evolutions in a single-stage system are also plotted for comparison. The values of the temporal and spectral shape factors and the phase factor at the output of the second stage are plotted as a function of the starting point of the second stage in the right graphs of Fig. 5. It can be seen that launching the pulses into the second fiber at the optimum $\xi$ relative to the first stage is not of crucial importance, especially with regard to the degree of parabolicity of the temporal profile of the output pulse. A closer inspection of Fig. 5 reveals that also the deviations of the spectral shape and phase factors from their optimum values are, nevertheless, very small. These results are confirmed by Fig. 6, which shows the pulse temporal and spectral intensity profiles and the chirp profile at the output of the second stage, when the second stage starts at the boundaries of the $\xi$-region considered in Fig. 5. It is worth noting here that starting the second stage beyond $\xi_{\text {opt }}$ leads to small oscillations in the far wings of the PP at the output of the second stage, which are an indication that the pulse will be subject to wave deformations on further propagation. However, still for a second stage starting point that is approximately twice as large as $\xi_{\text {opt }}$, such far-end oscillations in the output pulse from the two-stage device are very small, as it is seen from Fig. 6. The relatively large freedom in the choice of the initial reshaping fiber length for the generation of good-quality linearly-chirped PPs in a twostage fiber device suggested by the results in Figs. 5 and 6 can be particularly important for experimental implementations of the approach.

Next, we highlight the influence of the initial chirp on the PP generation process. Maps similar to those presented in Fig.
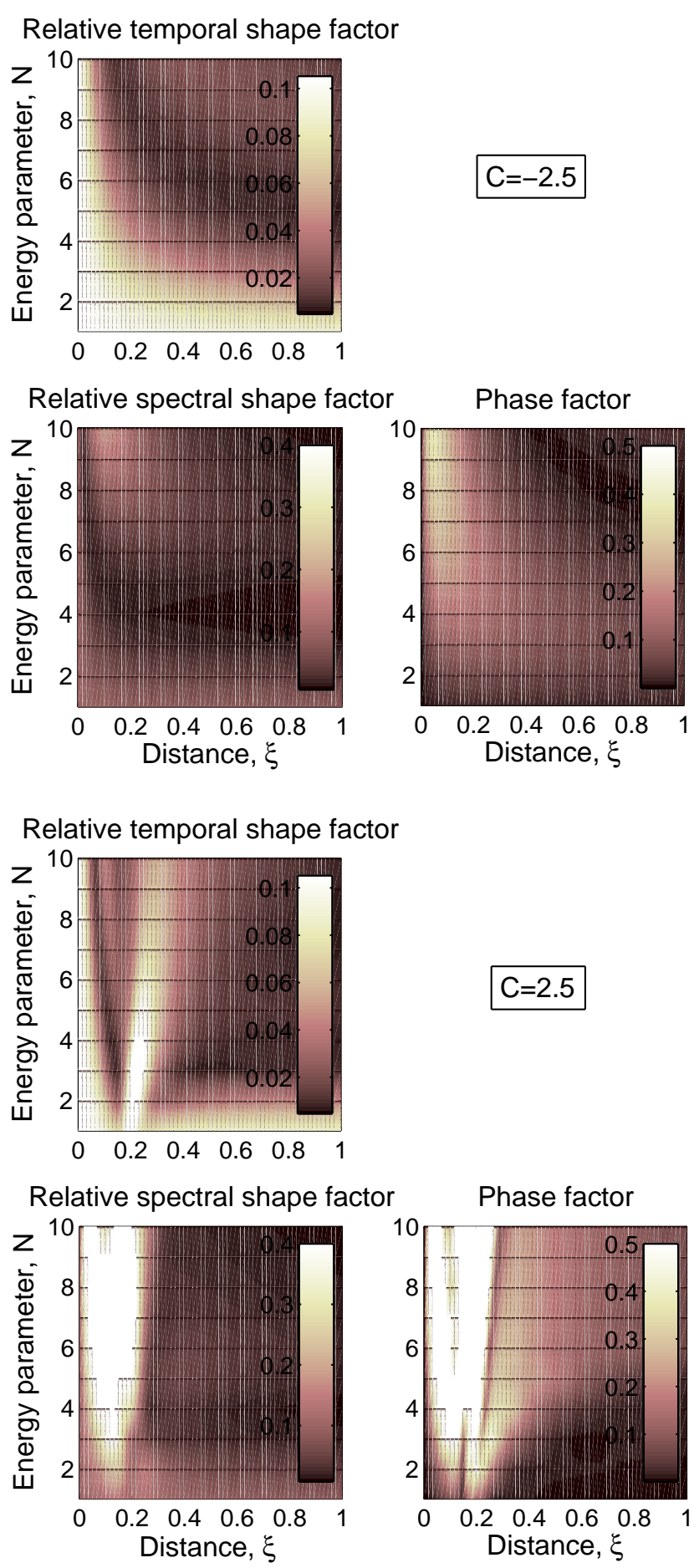

Fig. 7. First-stage evolution of the relative temporal and spectral shape factors $K$ and $\tilde{K}$, and the phase factor $\Phi$ (for $a=0.95$ ) versus $\xi$ and $N$ for $C=-2.5$ and $C=2.5$.

2 are plotted in Fig. 7 for a Gaussian pulse with $C=-2.5$ and $C=2.5$ used as the input. We observe that pulse reshaping is again obtained in the first fiber and that, just as for the case of an input chirp-free pulse, operational bands exist for which the output temporal intensity profile approaches the parabolic shape $(K \sim 0.01)$ and the frequency chirp is close to linear. Such bands correspond again to moderate values of the initial 


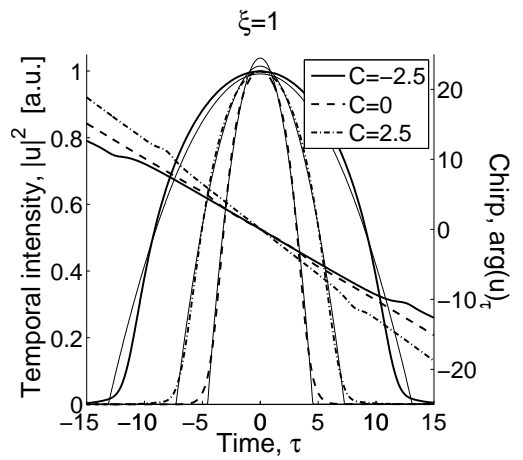

Fig. 8. Temporal intensity and chirp profiles at the output of the first stage for $C=-2.5(N=5), C=0(N=2.8)$, and $C=2.5(N=3)$. Thin solid lines: parabolic fits.

energy $(N \sim 4.5-6$ for $C=-2.5$ and $N \sim 3-4$ for $C=2.5)$. Parameter $K$ reaches its minimum $\left(k_{\text {opt }} \approx 0.0725\right)$ at $\left(N_{\text {opt }}=\right.$ $\left.5, \xi_{\text {opt }}=1\right)$ for $C=-2.5$, and at $\left(N_{\text {opt }}=3, \xi_{\text {opt }}=0.496\right)$ for $C=2.5$. We note that when $C<0$, the curves of the shape and phase factors for a given $N$ are monotonic in $\xi$. This is because the chirp parameter of an ideal PP is also negative. On the other hand, when $C>0$, the curves exhibit peaks and deeps, which indicate that the pulse is changing its chirp. More importantly, an initial non-zero chirp can stabilize the evolution of the temporal shape factor. We can observe that, although the minimum value of $K$ for $C=\mp 2.5$ is higher than that for the zero chirp, $K$ sets itself to a closeto-parabolic value $K \leq 0.01(\kappa \leq 0.0727)$ after the distance $\xi \approx 0.55$ for $C=-2.5$ and $\xi \approx 0.4$ for $C=2.5$ in Fig. 7 . The temporal intensity and chirp profiles at the output of the fiber $(\xi=1)$ for $C=\mp 2.5$ and $C=0$ are plotted in Fig. 8. Here, the corresponding optimum $N$ values are used. We note that the generated PP has a linear chirp with a negative slope as the ideal PP does, regardless of its initial chirp. Moreover, the intensity profiles illustrate how the initial chirp acts as a parameter (in addition to the initial energy) related with the width of the output PP.

The stabilization of the temporal shape factor in the first stage by an initial non-zero chirp observed in Fig. 7 is generally expected to lead to a higher tolerance of the characteristics of the pulses generated in the two-stage system to the length of the initial reshaping fiber compared with the case of a zero chirp. These expectations are confirmed by Fig. 9, which shows the temporal and spectral shape factors and the phase factor at the output of the second stage as a function of the second stage starting point $\xi$ for $C=\mp 2.5$ and $C=0$, and the corresponding optimum $N$ values. It is seen that an initial non-zero chirp yields a higher tolerance to the first fiber length in the spectral and phase domains irrespective of the chirp parameter sign, whereas in the temporal domain the tolerance for $C>0$ is slightly reduced compared with the case of $C=0$. However, we can see that the deviations of the temporal shape factor from its ideal parabolic value are very small for all chirp values. We also observe that launching an initially positively chirped pulse into the twostage device yields the best compromise between minimum achievable values of $\kappa, \tilde{\kappa}$ and $\Phi$ at the device output and

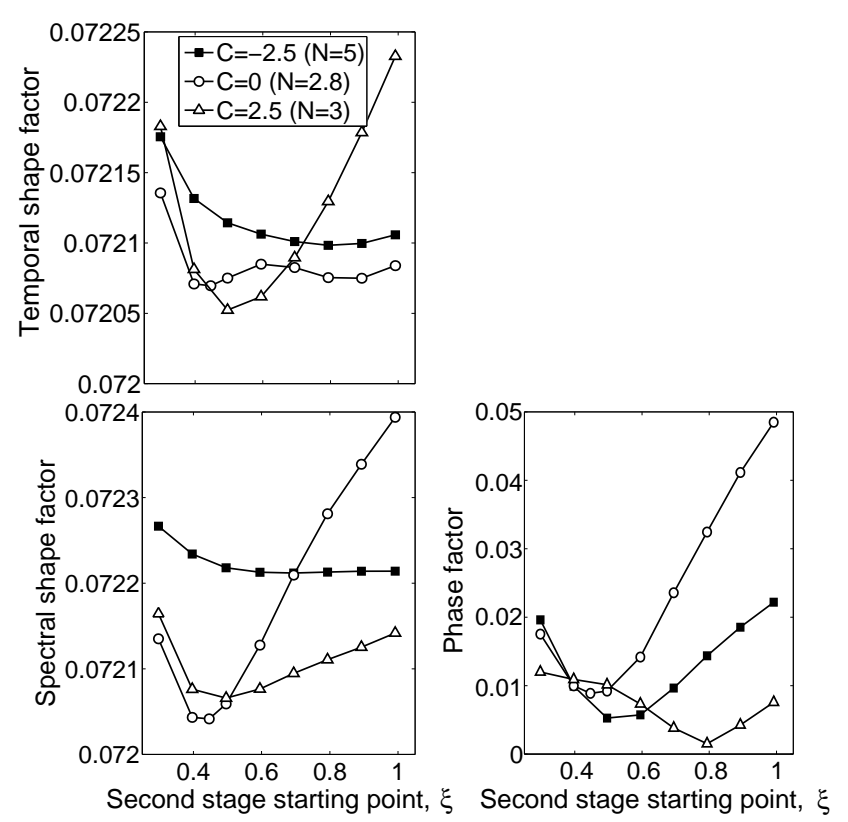

Fig. 9. Temporal and spectral intensity factors $\kappa$ and $\tilde{\kappa}$ and phase factor $\Phi$ at the output of the second stage as a function of the second stage starting point for $C=-2.5(N=5), C=0(N=2.8)$, and $C=2.5(N=3)$.

tolerance to the first fiber length. The results achieved here demonstrate that the initial chirp can be used to tailor the linearly chirped PPs generated in a fiber system, thus offering an additional degree of freedom. The chirp selection should be done in accordance with the available fibers.

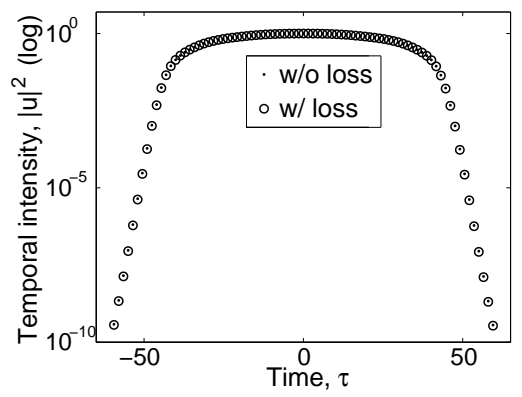

Fig. 10. Pulse temporal intensity profiles at the output of the two-stage device for the lossless and lossy systems $(\alpha=0.2 \mathrm{~dB} / \mathrm{km})$.

Finally, we briefly discuss the influence of fiber loss on the pulse reshaping process. It is well known that the loss effectively reduces the length $z$ of the fiber to a length $z_{\text {eff }}=$ $(1-\exp (-2 \Gamma z)) /(2 \Gamma)$, where $\Gamma=0.05 \ln (10) \alpha$ is the loss coefficient, and $\alpha$ is given in $\mathrm{dB} / \mathrm{km}$ [17]. A simple estimate for the typical value $\alpha=0.2 \mathrm{~dB} / \mathrm{km}$ of standard monomode fibers indicates that for the purpose of lossless propagation the fiber length should be $z \ll 1 / \Gamma \approx 40 \mathrm{~km}$. The effect of fiber loss remains negligible even for the more lossy HNL fibers with $\alpha \sim 1 \mathrm{~dB} / \mathrm{km}$ as long as $z$ does not exceed some hundreds of meters. In Fig. 10 we have plotted exemplary pulse temporal intensity profiles at the output of the two-stage fiber device for the lossless and lossy (with $\Gamma z \approx 0.01$ ) systems. It can be seen that the two curves are, indeed, indistinguishable. 


\section{NONLINEAR PULSE SHAPING IN A NORMALLY DISPERSIVE FIBER}

In Sec. III, we have seen that pulses with a parabolic intensity profile can be formed by passive nonlinear reshaping in a ND fiber. The best-quality parabolic pulses occur for moderate values of the initial pulse energy. Here, we demonstrate that using initial pulses with sufficiently high energy, rather different reshaping processes are possible in a short length of ND fiber.
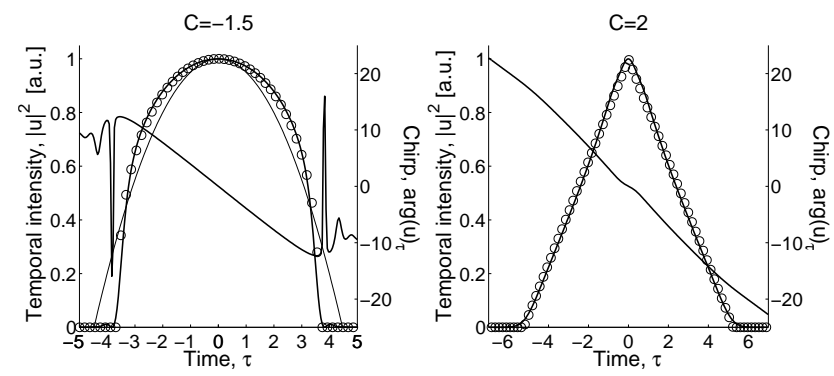

Fig. 11. Temporal intensity and chirp profiles in the ND fiber for $N=10$, and $C=-1.5$ at $\xi=0.215$ (left) and $C=2$ at $\xi=0.33$ (right). Solid curves: NLSE numerical simulation results, circles: corresponding fits. In the left graph, the parabolic fit (thin solid curve) is also shown.

We have observed that an initial, sufficiently powerful pulse can evolve towards a flat-top temporal shape for both zero and non-zero initial chirp and to a triangular shape when $C>0$ during propagation in a ND fiber. Both types of generated pulses have a negative chirp parameter similarly to the ideal PP. It is to be re-iterated that such pulse intensity profiles correspond to transient evolution regimes in the fiber. Figure 11 shows examples of the temporal intensity profiles and corresponding chirp profiles obtained using an initial chirped Gaussian pulse and $N=10$. The left graph in Fig. 11 shows a flat-top pulse generated for $C=-1.5$ at $\xi=0.215$. Such a pulse is characterized by a flatter top and steeper edges than a PP. The trial fitting function $\left|u_{\mathrm{R}}(\tau)\right|^{2}=\left[1-\left(\tau / \tau_{\mathrm{R}}\right)^{2}\right]^{b}$ if $|\tau| \leq \tau_{\mathrm{R}}$ and $\left|u_{\mathrm{R}}(\tau)\right|^{2}=0$ otherwise, is also plotted for $b=0.382$ and the same energy and peak power as those of the actual pulse, showing a good agreement with the actual pulse shape. This function yields $\kappa(b)=2 \Gamma(2 b)^{2} \Gamma(b+3 / 2)^{4} /\left(\pi b^{2}(b+3 / 2) \Gamma(b)^{4} \Gamma(2 b+3 / 2)^{2}\right)$, which matches the value of the generated pulse $(\kappa=0.0739)$ for $b=0.382$. The corresponding parabolic fit is also shown. Note that such a flat-top intensity profile corresponds to the evolution regime in the fiber immediately preceding wave breaking [1]. Function $\kappa(b)$ has the minimum at $b=1$, which corresponds to a parabolic intensity profile. A triangular pulse generated for $C=2$ at $\xi=0.33$ is shown in the right graph of Fig. 11. There is a good agreement between the actual intensity profile and the ideal triangular fit. In this example, $\kappa=0.0752$ for the generated pulse. Note that the triangular pulse shaping regime seems to be peculiar to the nonlinear evolution of an initial pulse with a positive chirp parameter in a ND fiber [17]. We can furthermore see from Fig. 11 that the frequency chirp exhibits a linear and monotonic behavior over the majority of the flat-top pulse profile and over the entire triangular pulse. Finally, we would like to point out that the shape factor $\kappa(\tilde{\kappa})$ used in this paper, which is defined solely in terms of the second-order moments related to the pulse temporal (spectral) width and power, does not characterize uniquely the shape of the pulse. Some information about the shape is still present and it is useful for gaining a physical insight into the pulse dynamics. More complete shape information could be provided by higher-order moments, which contain information in the time and frequency domains simultaneously.

\section{CONCLUSION}

We have presented an accurate characterization of the nonlinear reshaping of conventional pulses that occurs upon propagation in cascaded sections of commercially available ND fibers. We have analyzed in detail the evolution of an initial pulse into a pulse with a nearly parabolic temporal intensity profile and a nearly linear chirp during propagation in a ND fiber, and the subsequent pulse stabilization in a second ND fiber with specially adjusted nonlinear and dispersive characteristics relative to the first fiber. We have investigated the tolerance of the PP formation against variations in the length of the initial reshaping fiber as well as the influence of the initial pulse chirp on the pulse reshaping process. We have furthermore proposed the combination of pulse prechirping and propagation in a single section of ND fiber as a method for passive nonlinear pulse shaping, which provides a simple way of generating various pulse temporal shapes of fundamental and practical interest. We anticipate that the obtained results might be of great interest in the field of optical communications and signal processing, as well as in the field of pulsed lasers. We believe that our analysis, based on integral quantities (averaged over time or frequency) related to the main pulse characteristics, will stimulate the deployment of a larger array of mathematical tools to achieve a better description of the nonlinear pulse dynamics in ND fibers, which is becoming an active field of research.

\section{REFERENCES}

[1] D. Anderson, M. Desaix, M. Karlsson, M. Lisak, and M. L. QuirogaTeixeiro, "Wave-breaking-free pulses in nonlinear-optical fibers," J. Opt. Soc. Am. B, vol. 10, no. 7, pp. 1185-1190, 1993.

[2] K. Tamura and M. Nakazawa, "Pulse compression by nonlinear pulse evolution with reduced optical wave breaking in erbium-doped fiber amplifiers," Opt. Lett., vol. 21, no. 1, pp. 68-70, 1996.

[3] M. E. Fermann, V. I. Kruglov, B. C. Thomsen, J. M. Dudley, and J. D. Harvey, "Self-similar propagation and amplification of parabolic pulses in optical fibers," Phys. Rev. Lett., vol. 84, no. 26, pp. 6010-6013, 2000.

[4] S. Boscolo, S. K. Turitsyn, V. Yu. Novokshenov, and J. H. B. Nijhof, "Self-similar parabolic optical solitary waves," Theor. and Math. Phys., vol. 133, no. 3, pp. 1647-1656, 2002.

[5] C. Finot, S. Pitois, G. Millot, C. Billet, and J. M. Dudley, "Numerical and experimental study of parabolic pulses generated via Raman amplification in standard optical fibers," IEEE J. Sel. Top. Quantum Electron., vol. 10, no. 5, pp. 1211-1218, 2004.

[6] V. I. Kruglov and J. D. Harvey, "Asymptotically exact parabolic solutions of the generalized nonlinear Schrödinger equation with varying parameters," J. Opt. Soc. Am. B, vol. 23, no. 12, pp. 2541-2550, 2006.

[7] J. M. Dudley, C. Finot, G. Millot, and D. J. Richardson, "Self-similarity and scaling phenomena in nonlinear ultrafast optics," Nat. Phys., vol. 3, pp. 597-603, 2007.

[8] H. E. Lassen, F. Mengel, B. Tromborg, N. C. Albersten, and P. L. Christiansen, "Evolution of chirped pulses in nonlinear single-mode fibers," Opt. Lett., vol. 10, no. 1, pp. 34-36, 1985. 
[9] J. E. Rothenberg, "Femtosecond optical shocks and wave breaking in fiber propagation," J. Opt. Soc. Am. B, vol. 6, no. 12, pp. 2392-2401, 1989.

[10] T. Hirooka and M. Nakazawa, "Parabolic pulse generation by use of a dispersion-decreasing fiber with normal group-velocity dispersion," Opt. Lett., vol. 29, no. 5, pp. 498-500, 2004.

[11] A. Plocky, A. A. Sysoliatin, A. I. Latkin, V. F. Khopin, P. Harper, J. Harrison, and S. K. Turitsyn, "Experiments on the generation of parabolic pulses in waveguides with length-varying normal chromatic dispersion," JETP Lett., vol. 85, no. 7, pp. 319-322, 2007.

[12] B. Kibler, C. Billet, P. A. Lacourt, R. Ferrière, L. Larger, and J. M. Dudley, "Parabolic pulse generation in comb-like profiled dispersion decreasing fibre," Electron. Lett., vol. 42, no. 17, pp. 965-966, 2006.

[13] A. I. Latkin, S. K. Turitsyn, and A. A. Sysoliatin, "Theory of parabolic pulse generation in tapered fiber," Opt. Lett., vol. 32, no. 4, pp. 331-333, 2007.

[14] F. Parmigiani, C. Finot, K. Mukasa, M. Ibsen, M. A. F. Roelens, P. Petropoulos, and D. J. Richardson, "Ultra-flat SPM-broadened spectra in highly nonlinear fiber using parabolic pulses formed in a fiber Bragg grating," Opt. Express, vol. 14, no. 17, pp. 7617-7622, 2006.

[15] C. Finot, L. Provost, P. Petropoulos, and D. J. Richardson, "Parabolic pulse generation through passive nonlinear pulse reshaping in a normally dispersive two segment fiber device," Opt. Express, vol. 15, no. 3, pp. 852-864, 2007.

[16] Y. Park, M. H. Asghari, T. -J. Ahn, and J. Azaña, “Transform-limited picosecond pulse shaping based on temporal coherence synthesization," Opt. Express, vol. 15, no. 15, pp. 9584-9599, 2007.

[17] G. P. Agrawal, Nonlinear Fiber Optics (3rd ed., Academic Press, 2001).

[18] A. I. Latkin and S. K. Turitsyn, "Semi-analytical description of parabolic pulse generation in the normal-dispersion fibre amplifiers," in Proc. of the 8th International Conference on Transparent Optical Networks (ICTON 2006), pp. 259-262, Nottingham, United Kingdom, Jun. 2006.

[19] B. Burgoyne, N. Godbout, and S. Lacroix, "Nonlinear pulse propagation in optical fibers using second order moments," Opt. Express, vol. 15, no. 16, pp. 10075-10090, 2007.

[20] E. G. Shapiro and S. K. Turitsyn, "Theory of guiding-center breathing soliton propagation in optical communication systems with strong dispersion management," Opt. Lett., vol. 22, no. 20, pp. 1544-1546, 1997.

Sonia Boscolo was born in Turin, Italy. She received the B.Sc. and M.Sc. degrees in physics from the University of Burgundy, Dijon, France, in 1998, and the Ph.D. degree in engineering and applied science from Aston University, Birmingham, U.K., in 2002.

Since 2002, she has been working with the Photonics Research Group, School of Engineering and Applied Science, Aston University, where she is a Senior Research Fellow.

Dr. Boscolo was awarded a Leverhulme Trust Early Career Fellowship in 2005, and a full three-year EPSRC grant in 2007.

Anton I. Latkin graduated from the Department of Physics, Novosibirsk University, Novosibirsk, Russia, in 2004, and received the Ph.D. degree in optics from the Institute of Automation and Electrometry, Novosibirsk, Russia, in 2007.

He is a Research Fellow at the Novosibirsk State University and a visiting scholar at Aston University, Birmingham, U.K.

Sergei K. Turitsyn graduated from the Department of Physics, Novosibirsk University, Novosibirsk, Russia, in 1982, and received the Ph.D. degree in theoretical and mathematical physics from the Institute of Nuclear Physics, Novosibirsk, Russia, in 1986.

From 1992 to 1998 , he was with the Institute for Theoretical Physics I, Heinrich-Heine University, Duesseldorf, Germany, first as a Humboldt Fellow, and later as a leader of the collaborative projects with Deutsche Telekom. He joined the Photonics Research Group, Aston University, Birmingham, U.K., in 1998, where he is a Professor in the School of Engineering and Applied Science and leads the Theory and Modelling Group. 